\title{
Validation Metrics for Non-Rigid Registration of Medical Images containing Vessel Trees
}

\author{
Thomas Lange ${ }^{1}$, Hans Lamecker ${ }^{2}$, Michael Hünerbein ${ }^{1}$, Sebastian Eulenstein ${ }^{1}$, \\ Siegfried Beller ${ }^{1}$, Peter M. Schlag ${ }^{1}$ \\ ${ }^{1}$ Department of Surgery and Surgical Oncology, Charité - Universitätsmedizin Berlin \\ ${ }^{2}$ Zuse Institute Berlin \\ thomas.lange@charite.de
}

\begin{abstract}
Validation of non-rigid registration methods is still a challenging task. Different evaluation criteria were published, yet no widely accepted gold standard exists. The aim of this paper is to provide quantitative evaluation metrics suited for clinical 3D images containing vessel trees, such as liver or brain data. We present a method to identify corresponding points on different vessel trees by extracting consistent graph minors interactively. Four different metrics based on these correspondencies are proposed.
\end{abstract}

\section{Introduction}

Validation of non-rigid registration approaches is a difficult problem. While an accepted gold standard exists for validation of rigid registration methods[1] only few papers address this issue for non-rigid registration $[2,3]$. The published methods are based on simulated data or physical phantoms [4] on the one hand and a restriction of the validation to some important anatomical structures that can be identified in real clinical data on the other hand. The restricted validation on anatomical structures can be based on richly annotated (labeled, segmented) real images [3] or interactively assigned corresponding anatomical landmarks [5].

Since it is challenging to generate simulated data or physical phantoms, possessing realistic imaging and deformation properties, we focus on restricted validation methods for real clinical data. Often vessel trees are suitable anatomical structures for registration validation in particular in liver and neurosurgery.

In a first step corresponding vessel branchings in both modalities are identified interactively. Then an automatic algorithm determines corresponding substructures (graph minors) of the vessel trees while verifying their consistency. Four evaluation metrics are defined on those substructures. To validate a nonrigid registration result the vessels in the model data are deformed with the computed transformation and the metrics measure how close corresponding vessel parts in the reference data are after the transformation. 


\section{Methods}

It is assumed, that a segmentation and center line extraction (via skeletonization) of the vessel trees from two different data sets (e.g. CT and 3D ultrasound) exists. In our case the center lines consist of linearly interpolated 3D node point sequences. Based on those nodes the vessels can be represented as a tree (Fig 1a). Let $T=(V, E)$ and $\hat{T}=(\hat{V}, \hat{E})$ be directed trees for the reference resp. model data. All edges point in the direction to the given root nodes $r \in V$ and $\hat{r} \in \hat{V}$. The node subsets $B \subseteq V$ and $\hat{B} \subseteq \hat{V}$ contain all branching nodes (node degree $\delta(v)>2)$. Let $P(v, w)$ be the unique path from node $v$ to node $w$. We call a path between two branching nodes or a branching and an end node $(\delta(v)=1)$ a (vessel) segment path.

The trees $T, \hat{T}$ are not identical and only parts of the underlying anatomical vessel tree (supertree) can be identified in both modalities. There is no subtree isomorphism of $T, \hat{T}$ to a common supertree and even no homeomorphism. Due to missing branches a segment path in $T$ might be represented by several segment paths in $\hat{T}$ and vice versa (Fig. 1a). In addition the order of branching points or other topological changes might occur (Fig.1b) caused by inaccuracies in the image processing pipeline. To get a subtree isomorphism the contraction of some segment paths to one single node is necessary. This means a supertree contains $T$ and $\hat{T}$ as a minor. Intuitively, a graph $G$ is a minor of a graph $H$, if $G$ can be obtained from $H$ by a series of vertex/edge deletions and edge contractions.

As the consistent assignment of branching nodes and segment paths is only heuristically solvable based on geometric properties [6], we interactively define a subset of corresponding branching points $\left(b_{i}, \hat{b}_{i}\right), i=1, \ldots, n, b_{i} \in B, \hat{b}_{i} \in \hat{B}$. Let $B_{c o r r}, \hat{B}_{c o r r}$ contain all $b_{i}$ resp. $\hat{b}_{i}$. To ease the interaction, a mouse click can be set onto the vessel surface nearby the branching point and the nearest branching point on the center line graph is determined automatically.

\subsection{Determination of corresponding segment paths}

Algorithm 1 determines for two given vessel center line trees $T, \hat{T}$ and a subset of corresponding branching node pairs $\left(b_{i}, \hat{b}_{i}\right), i=1 \ldots n$ corresponding path pairs

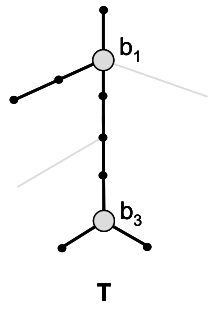

a)

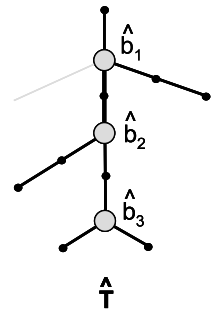

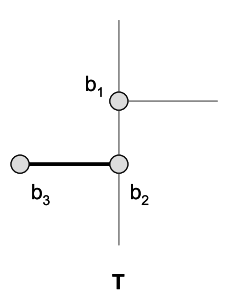

b)

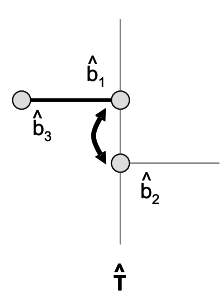

Fig. 1. a) Reference $T$ and model tree $\hat{T}$ are minors of the underlying anatomical tree (grey). b) Caused by inaccuracies the order of the branching nodes might be exchanged. 
$\left(P_{i}, \hat{P}_{i}\right)$. As the directed path to the root is unique, the algorithm starts from each corresponding branching point and ends, if another corresponding branching point or a point, which has already been visited, has been reached. The second termination criterion is necessary because not all branching points might have been interactively assigned. It is important to check if the reached points are an assigned branching point pair in order to detect topological or assignment errors. Most of the inconsistencies can be resolved by automatic contraction of very

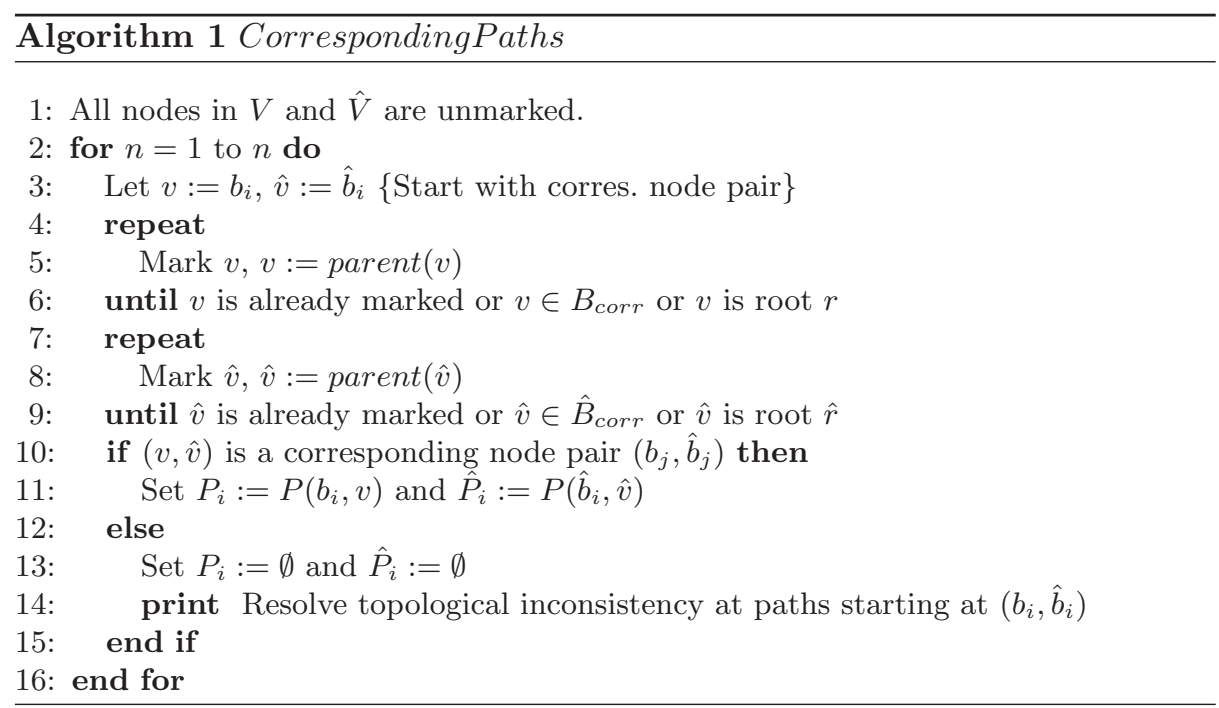

short segment paths. Remaining problems are eliminated interactively. Based on the resulting path pairs each model path $\hat{P}_{i}$ is reparameterized according to the reference path $P_{i}$. This means the relative distances between successive reference nodes are transfered to the length of the model path. Now each node on a reference line corresponds to a point on the model line. Let $\left(p_{j}, \hat{p_{j}}\right), j=1, \ldots, m$ be all corresponding point pairs on all segments.

\subsection{Evaluation metrics}

Distance of corresponding points on center lines: The most simple metric is the average distance between corresponding point pairs.

$$
M_{\text {dist }}(T, \hat{T}):=\frac{1}{m} \sum_{j=1}^{m}\left\|p_{j}-\hat{p}_{j}\right\|
$$

Weighted point distance: The corresponding center line points are generated with some location uncertainties. The uncertainties along the lines are usually higher than perpendicular to the lines. In particular location uncertainties of the 
branching points lead to translations of the correspondences along the lines. Let $\Sigma_{j}$ be an estimated covariance matrix of the anisotropic location uncertainty for the point pair $\left(p_{j}, \hat{p}_{j}\right)$. Based on these weighting matrices a weighted distance measure can be defined [7]:

$$
M_{\text {weighted }}(T, \hat{T}):=\frac{1}{m} \sum_{j=1}^{m}\left(p_{j}-\hat{p}_{j}\right)^{T} \Sigma_{j}^{-1}\left(p_{j}-\hat{p}_{j}\right)
$$

Directional deviation metric: From the vessel center lines in each corresponding point pair $\left(p_{j}, \hat{p}_{j}\right)$ (except in the branching points) a normalized tangential vector pair $\left(d_{j}, \hat{d}_{j}\right)$ can be computed. The sine of the angle between the direction vectors is a measure for the deviation of the two directions. As the cross product $(x)$ is related to the sine an evaluation metric can be defined by:

$$
M_{d i r}(T, \hat{T})=\frac{1}{m} \sum_{j=1}^{m}\left\|d_{j} \times \hat{d}_{j}\right\|
$$

Segment-wise relative volume overlap: A metric that is less dependent on the center line extraction is the relative volume overlap of corresponding vessel segments. Each voxel is assigned to the closest segment path point. For all points on corresponding segment paths $P_{i}, \hat{P}_{i}$ the assigned voxels define the volumes $V_{i}, \hat{V}_{i}$ of the particular segments.

$$
M_{v o l}(T, \hat{T}):=\frac{1}{n} \sum_{i=1}^{n} \frac{V_{i} \cap W_{i}}{V_{i} \cup W_{i}}
$$

\section{Results}

The corresponding path algorithm has been exemplarily applied to the portal veins extracted from preoperative contrast-enhanced CT (Fig. 2a) and intraop-
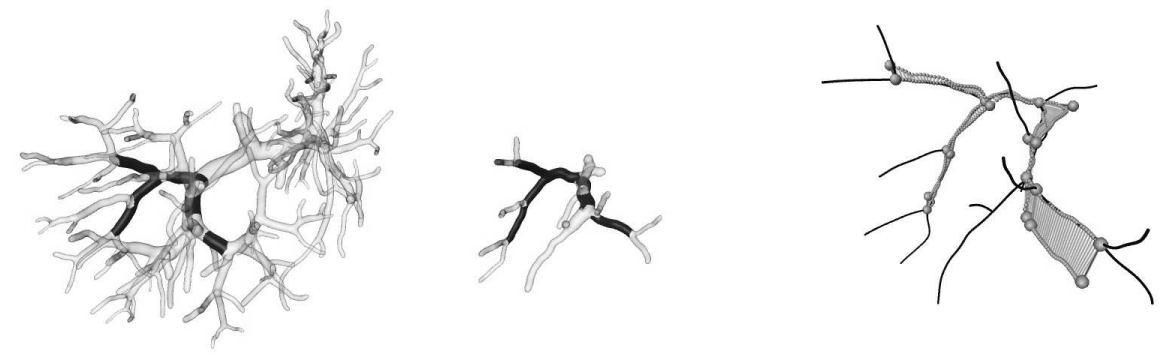

Fig. 2. a) Portal veins extracted from contrast-enhanced CT data and b) from 3D Powerdoppler ultrasound data (black: corresponding vessel segments). c) Vessel center lines from ultrasound data (black) and corresponding points in CT data (small spheres and grey lines) determined from corresp. branching nodes (big spheres). 
erative 3D powerdoppler ultrasound data (Fig. 2b). Based on the corresponding center line point pairs a rigid transformation was computed. The remaining deviations of the vessels are illustrated in Fig. 2c) and are on average $4.5 \mathrm{~mm}$.

\section{Discussion and Future Work}

In computer assisted liver surgery one important task is to non-rigidly register preoperative 3D models of anatomical structures to intraoperative 3D ultrasound data $[8,9]$ which are the reference data for navigation systems in liver surgery [10]. We introduced validation methods for the accuracy of non-rigid registration approaches on image pairs containing vessel trees. Each validation metric stresses a different aspect of registration accuracy. The metrics can be applied for registration validation of preoperative $\mathrm{CT}$ with intraoperative ultrasound data or pre- and postoperative CT data of the liver for follow-up studies. The aim is to generate a database of corresponding segment paths for portal and hepatic veins from 20-25 cases for each of the two applications. One important future task is to improve the precision of the center line extraction and to investigate the remaining uncertainties of the correspondences more rigorously. In particular the segmentation of the vessels might be improved by fitting local vessel models.

\section{References}

1. West J, Fitzpatrick JM, Wang MY, et al. Comparison and evaluation of retrospective intermodality brain image registration techniques. J Comput Assist Tomogr. 1997;21(4):554-66.

2. Hellier P, Barillot C, Corouge I, et al. Retrospective evaluation of inter-subject brain registration. Proc MICCAI. 2001; p. 258-65.

3. Christensen G, Geng X, Kuhl J, et al. Introduction to the non-rigid image registration evaluation project (NIREP). Proc WBIR. 2006; p. 128-35.

4. Reinertsen I, Descoteaux M, Siddiqi K, et al. Validation of vessel-based registration for correction of brain shift. Med Image Anal. 2007;11(4):374-88.

5. Reinertsen I, Lindseth F, Unsgaard G, et al. Clinical validation of vessel-based registration for correction of brain-shift. Med Image Anal. 2007;11(6):673-84.

6. Charnoz A, Agnus V, Malandain G, et al. Design of robust vascular tree matching: Validation on liver. Proc IPMI. 2005; p. 443-55.

7. Rohr K, Stiehl H, Sprengel R, et al. Landmark-Based Elastic Registration Using Approximating Thin-Plate Splines. IEEE Trans Med Imaging. 2001;20(6):526-34.

8. Lange T, Eulenstein S, Hünerbein M, et al. Augmenting intraoperative 3D ultrasound with preoperative models for navigation in liver surgery. Lect Notes Comp Sci. 2004;3217:534-41.

9. Lange $\mathrm{T}$, Lamecker H, Hünerbein M, et al. A new class of distance measures for registration of tubular models to image data. Proc BVM. 2007; p. 101-5.

10. Beller S, Hünerbein M, Eulenstein S, et al. Feasibility of navigated resection of liver tumors using multiplanar visualization of intraoperative 3D ultrasound data. Annal Surg. 2007;246(2):288-94. 